\title{
Case report of a rare glomus jugulare tumor extended within the extracranial internal jugular vein: emphasis on initial ultrasound findings and a literature review
}

\author{
Qing Deng, Yi-Jia Wang, Xiao-Yi Chen, Qing Zhou
}

Department of Ultrasound Imaging, Renmin Hospital of Wuhan University, Wuhan, China

\begin{abstract}
Glomus jugulare tumor (GJT) is a rare benign neoplasm that is located in the skull base; it is impossible for ultrasonography to detect it in most cases. In this article, we present the case of a rare giant GJT, which extended into the internal jugular vein in the neck, initially detected by ultrasonography and confirmed by MRI and pathology. We highlight the initial ultrasonography findings and review the literature to summarize the ultrasonographic features of GJT.
\end{abstract}

Keywords: glomus jugulare tumor; ultrasonography; MRI

\section{Introduction}

Glomus jugulare tumor (GJT), a rare benign tumor, is a paraganglioma of the head and neck that is confined to the jugular fossa [1]. Although mostly benign and slowgrowing, GJT has a propensity for local invasion of adjacent structures. The initial clinical symptoms of GJT are scarce, so delays in diagnosis are frequent [2-3]. Radiological imaging techniques, especially magnetic resonance imaging (MRI), are the major methods for the diagnosis of GJT. There are very few reports of ultrasound in the diagnosis of GJT, the GJT being located in the skull base [4-5]. We present the case of a rare giant GJT, which extended within the internal jugular vein (IJV) and was detected by ultrasonography. We highlight the initial ultrasonographic findings of GJT and revised relevant literature.

\section{Case report}

A 61-year-old woman presented with a painless and gradually enlarging mass in her left neck

Received 31.07.2017 Accepted 23.08.2017

Med Ultrason

2018, Vol. 20, No 1, 111-113

Corresponding author: Qing Zhou

238\# Jiefang Road, Wuchang District,

Wuhan City, Hubei, China, 430060

Phone: +86 13971358226, Fax: +86 2788040334

E-mail: qingzhou128@163.com for two years. The previous month, she had felt pulsatile tinnitus, dizziness, and hearing loss. A high frequency ultrasonography was performed and the ultrasound imaging revealed a giant hypoechoic mass in the left IJV. The mass was dumbbell-shaped and occupied the whole left IJV. The mass (more than $14 \mathrm{~cm}$ in length) was immobile and no erosions of the venous wall were detected. The upper end of the mass could not be found because it originated from the jugular foramen. The width of the mass was from $0.8 \mathrm{~cm}$ to $3.2 \mathrm{~cm}$ and the thinnest part was located in the valve of the IJV. Color Doppler flow imaging showed rich blood flow signals inside the mass with a downward direction. Pulse wave Doppler demonstrated that it was arterial blood flow inside the mass with a low resistance index (fig 1). All of these ultrasonographic findings indicated the mass was a solid tumor with a rich blood supply.

The following high-resolution computer tomography (CT) scan showed a large soft tissue density mass in the left neck with local bone destruction in skull base. MRI showed that the high T2 signal of the tumor and the multiple flow voids formed mixed signals, which were described as "salt and pepper" appearance [6] (fig 2). The final radiologic impression of the mass was a glomus jugulare tumor.

The patient finally underwent resection. During operation, the surgeons found the mass did not erode the 

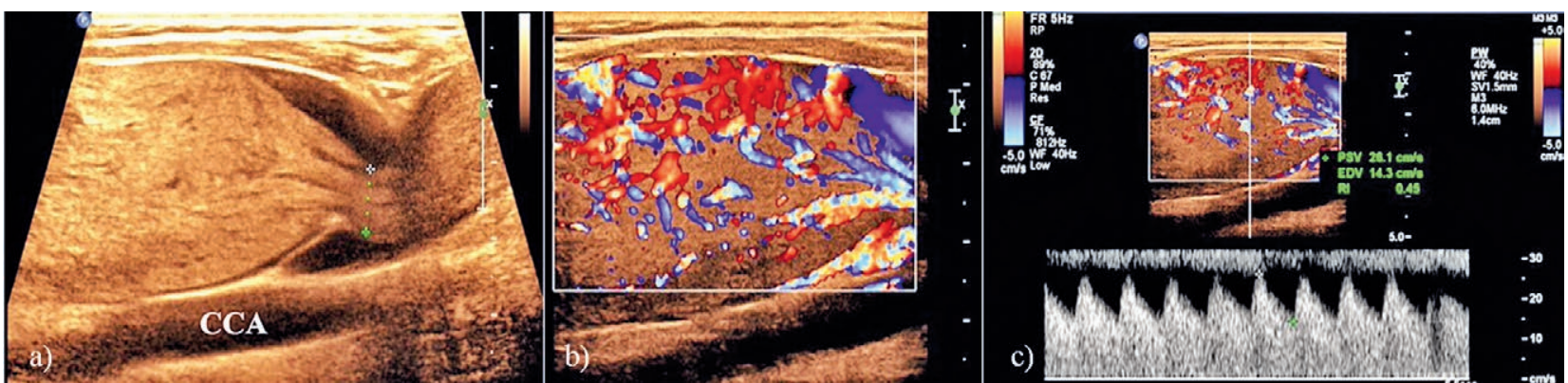

Fig 1. Ultrasound imaging of the glomus jugulare tumor. A giant dumbbell-shaped solid mass in the internal jugular vein (a) with rich blood flow signals (b) and low resistance index (c).
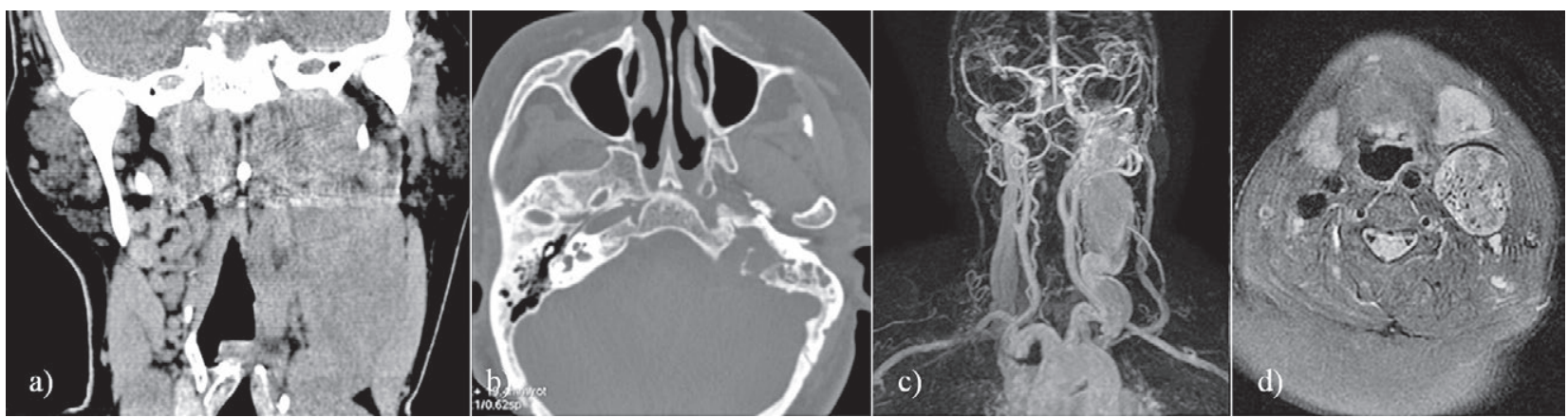

Fig 2. CT scan evidenced a large soft tissue density mass in the left neck with local bone destruction in tympanum (a,b). MRI showed the mass originated from the left jugular foramen (c) and formed the characteristic "salt and pepper" sign (d).

venous wall of IJV. The mass was completely excised. Pathological diagnosis verified the mass was a GJT.

\section{Discussions}

GJT is also referred to as paraganglioma or a chemical receptor tumor. It has a female dominance of about $3: 1$ and mainly afflicts people in midlife [2,7]. The most common symptoms of GJT are tinnitus and aural pulsations, dizziness, decreased hearing. The initial clinical presentations are insidious even if GJT reach a large size. Accurate and comprehensive imaging evaluation is the key for the diagnosis of GJT. Because the mass is located in the skull base and ultrasound cannot penetrate the bone, GJT is most commonly found by other imaging techniques [4-5]. In our case the GJT was giant size, located in the jugular foramen, and extended inferiorly to the end of the IJV - these were the reasons why it was clearly detected by ultrasonography.

Despite the fact that by ultrasound the origin of the mass could not be evaluated, we considered it may be a benign tumor, originating from the base of skull. There were three reasons that explained our consideration. First, the mass was top-down growing in the IJV but we could not find the origin of the mass in the extracranial
IJV. Second, the artery blood flow signals inside the mass are downward and consistent with a low resistance index. Third, the mass had not eroded the wall of the IJV. It was interpreted as an important imaging manifestation reflecting the soft and benign biological behaviour of the tumor. The following radiological imaging and pathology verified the mass was a GJT.

Due to the rarity of a giant GJT extended into the IJV, at the time of this manuscript writing, our search of the English language literature revealed only 2 individual cases reporting ultrasonographic findings of GJT $[8,9]$. Until now, the characteristic ultrasonographic appearance of GJT has not been systematically summarized. We revised the previous reports and summarized the ultrasonographic findings of GJT. In general, GJT displayed as a hypoechoic mass elongated into the IJV from the jugular foramen. The mass does not invade the venous wall though it has reached a large size. There were rich blood supplies in the mass and the blood flows are downwardly directed. These findings were displayed in all 3 cases.

The most common mass within the IJV is thrombus. Ultrasonography can easily differentiate a tumor from thrombus because there is no blood flow in the latter. Primary tumors of the IJV are extremely rare and ultra- 
sonography can show the relation between tumors and IJV walls. Tumors in the IJV almost always originate from cervical soft tissue and head. Tumors that originate from cervical soft tissue usually invade the wall of the IJV. For tumors that originate from intracranial tissue, it is hard for ultrasonography to determine their origin, but the elongated mass into the IJV from the jugular foramen can be found. The most common type of jugular foramen tumor elongated into the IJV are meningioma and schwannoma. Hypervascularity has been considered to be a helpful criterion for establishing the diagnosis of a GJT by ultrasonography [8]. MRI is much more reliable in the differential diagnosis of jugular foramen tumors. MRI can clearly show the intracranial and extracranial parts of the tumor and the surrounding tissues. In MRI images, the high signal of the tumor and the low signal of the multiple flow voids form so-called "salt and pepper" sign, which is the characteristic appearance of GJT. Schwannoma usually has a characteristic well circumscribed, elongated configuration following the course of the cranial nerves of origin and the rat tail sign refers to the characteristic appearance of meningioma. These characteristic appearances make MRI often able to identify these tumors before operation [10].

In conclusion, GJTs extended into IJV are rare and ultrasonography is usually the initial method used to detect these masses. Ultrasonography can easily differentiate them from thrombus and cervical soft tissue tumors but it is hard for ultrasonography to determine their origin. MRI is helpful in reaching the correct diagnosis before operation.

\section{References}

1. Hafez RF, Morgan MS, Fahmy OM. An intermediate term benefits and complications of gamma knife surgery in management of glomus jugulare tumor. World J Surg Oncol 2016;14:36.

2. Michael LM 2nd, Robertson JH. Glomus jugulare tumors: historical overview of the management of this disease. Neurosurg Focus 2004;17:E1.

3. Carlson ML, Sweeney AD, Wanna GB, Netterville JL, Haynes DS. Natural history of glomus jugulare: a review of 16 tumors managed with primary observation. Otolaryngol Head Neck Surg 2015;152:98-105.

4. van den Berg R. Imaging and management of head and neck paragangliomas. Eur Radiol 2005;15:1310-1318.

5. Moore MG, Netterville JL, Mendenhall WM, Isaacson B, Nussenbaum B. Head and neck paragangliomas: an update on evaluation and management. Otolaryngol Head Neck Surg 2016;154:597-605.

6. Lee KY, Oh YW, Noh HJ, et al. Extraadrenal paragangliomas of the body: imaging features. AJR Am J Roentgenol 2006; 187:492-504.

7. Woolen S, Gemmete JJ. Paragangliomas of the Head and Neck. Neuroimaging Clin N Am 2016;26:259-278.

8. Alkadhi H, Schuknecht B, Stoeckli SJ, Valavanis A. Evaluation of topography and vascularization of cervical paragangliomas by magnetic resonance imaging and color duplex sonography. Neuroradiology 2002;44:83-90.

9. Qi ZH, Jiang YX, Feng F, Dai Q. Ultrasound characteristics of a glomus jugulare tumor. J Neuroimaging 2013;23:242244.

10. Chen ZC, Wang CP, Hsiao JK, Ko JY, Tseng HM, Yao YT. Angiomatous type of jugular foramen meningioma with neck extension: differential diagnosis from paraganglioma and schwannoma. Head Neck 2007;29:793-798. 